\title{
Anaesthetic Management of an Achondroplastic Dwarf with Difficult Airway and Spine for Total Hip Replacement: A Case Report
}

Neisevilie Nisa ${ }^{*}$, Puneet Khanna and Dhruv Jain

Department of Anesthesia, Critical Care and Pain Medicine, All India Institute of Medical Sciences, New Delhi, India

*Corresponding author: Neisevilie Nisa, Department of Anesthesia, Critical Care and Pain Medicine, All India Institute of Medical Sciences, New Delhi 110029, India, Tel: 08376960134; E-mail: neisevilie@gmail.com

Rec date:February 24, 2016; Acc date: March 30, 2016; Pub date: April 1, 2016

Copyright: (c) 2016 Nisa N, et al. This is an open-access article distributed under the terms of the Creative Commons Attribution License, which permits unrestricted use, distribution, and reproduction in any medium, provided the original author and source are credited.

\begin{abstract}
Achondroplasia is the commonest form of dwarfism and possesses multiple anesthetic challenges ranging from securing intravenous line, monitoring and calculating drug dosage to abnormalities of the airway, spine, thoracic cage, and obesity, cardiopulmonary and neurological system. Multiple case reports describing neuraxial anaesthesia, particularly in obstetrics patients are available but there are limited literature on airway management and general anaesthesia. We came across an achondroplastic dwarf for hip replacement with spine deformity where neuraxial anaesthesia was impossible and her difficult airway and chronic lung disease imposed serious anesthetic challenges. We describe the importance of awake fiberoptic intubation (FOI) for airway management and general anaesthesia in this patient with difficult airway and spine and restrictive lung disease for total hip replacement.
\end{abstract}

Keywords: Achondroplasia; Awake fiberoptic intubation

\section{Key Messages}

Achondroplastic dwarf presents with airway changes, restrictive lung disease and vertebral deformity imposing significant challenges for both general and neuraxial anaesthesia. Thorough knowledge and proper planning is required to avoid airway catastrophe and other anaesthesia related complications while administering anaesthesia in these patients.

\section{Introduction}

Achondroplastic dwarfism is the commonest form of skeletal dysplasia with an incidence of 4-15 per 100,000 live births with female affected more then males $[1,2]$. It has an autosominal dominant trait with $90 \%$ sporadic and $10 \%$ familial mutation due to G-to-A substitution in fibroblast growth factor receptor 3(FGFR-3) located on nucleotide 1138 resulting in decreased endochondral ossification [2,3]. They post unique anesthetic challenges due to the involvement of multiple organ system. We discuss the anesthetic management of such a patient with difficult airway and spine with severe pulmonary disease and obesity for bilateral total hip replacement.

\section{Case History}

A 59 year old female achondroplastic dwarf with bronchial asthma for past 20 years with steroid induced avascular necrosis of femur was posted for bilateral total hip replacement (THR). She stands $121 \mathrm{~cm}$ tall and weighed $48 \mathrm{~kg}(\mathrm{BMI}=32.83)$ with features of dwarfism. Her airway assessment revealed a large head and occiput, depressed nose, large tongue with receding mandible, short neck with circumference of $41.68 \mathrm{~cm}$ and marked limitation of extension, decreased submandibular distance $(4.2 \mathrm{~cm})$ and large breast. Cardiopulmonary examination revealed wheezing over multiple lung fields. Chest $\mathrm{x}$-ray suggested small airway disease with hyperinflated lung and increase vascular markings. Spine MRI showed dorsal and lumbar kyphoscoliosis with degenerative changes and fusions of all dorsal and lumbar spines. There was also narrowing of the foramen magnum and fusion of C3-C5 cervical vertebral bodies which limited neck extension.

Pulmonary function test (PFT) revealed severe restrictive and obstructive lung disease with FEV1 $=0.26 \mathrm{~L}(18 \%), \mathrm{FVC}=0.35 \mathrm{~L}(20 \%)$ $($ ratio $=89)$; after bronchodilator FEV1 $=0.64 \mathrm{~L}(36 \%), \mathrm{FVC}=0.39 \mathrm{~L}$ $(27 \%)($ ratio $=76)$. Other laboratory parameters were unremarkable and after one week of chest physiotherapy she was posted for surgery. In view of her vertebral abnormality, cervical spine instability and expected difficult airway, awake fiberoptic intubation and general anaesthesia with control ventilation was planned.

On the day of the surgery, injection glycopyrrolate $200 \mu \mathrm{g}$ intramascular was given 30 minutes before shifting to the operation room (OR) and four drops of xylometazoline $(0.05 \%)$ were given in both nostrils in the OR. Difficult airway cart was prepared and toxic local anesthetic dose was calculated. A 20 gauge peripheral intravenous (IV) line was secured after multiple attempts. Airway preparation was done by nebulization with $4 \mathrm{ml}$ of $4 \%$ lignocaine for 10 minutes followed by spraying of the base of tongue and posterior pharyngeal wall with $10 \%$ lignocaine. Awake fiberoptic nasal intubation was done using spray as you go technique maintaining cervical stability throughout and intubation was successful in the first attempt. After securing the airway, intravenous induction was done and patient was put on mechanical ventilation. Right radial artery was cannulated for invasive blood monitoring and an additional peripheral IV line was secured and surgery was allowed to begin. The intraoperative blood loss was more than usual and the patient became hemodynamically unstable. The surgical team decided to do only unilateral THR due to the sudden blood loss which has resulted in hemodynamic unstability. Patient was not extubated due to her poor chest condition preoperatively, difficult intubation and increase blood loss and was shifted to ICU for postoperative mechanical ventilation and monitoring. 
Postoperative analgesia included intravenous fentanyl infusion and paracetamol maintaining a visual analogue score (VAS) of less than 4. Trachea was extubated 14 hours later when she was fully awake. Patient had an uneventful post-operative period and underwent THR of the opposite side after one week and was discharged 5 days later.

\section{Discussion}

Achondroplastic dwarf presents with numerous anaesthetic challenges and careful preoperative evaluation is required for optimization and documentation of the comorbid conditions to have a productive post-operative outcome.

Difficult airway features (Figure 1) are present due to the premature fusion of the skull base, frontal bossing, depressed nasal bridge, maxillary hypoplasia, macroglossia, abnormal teeth implantation, large mandible, short neck with fat deposition, cervical osteophytes and anomaly causing atlanto-axial instability and limited neck extension $[1,3,4,5]$.

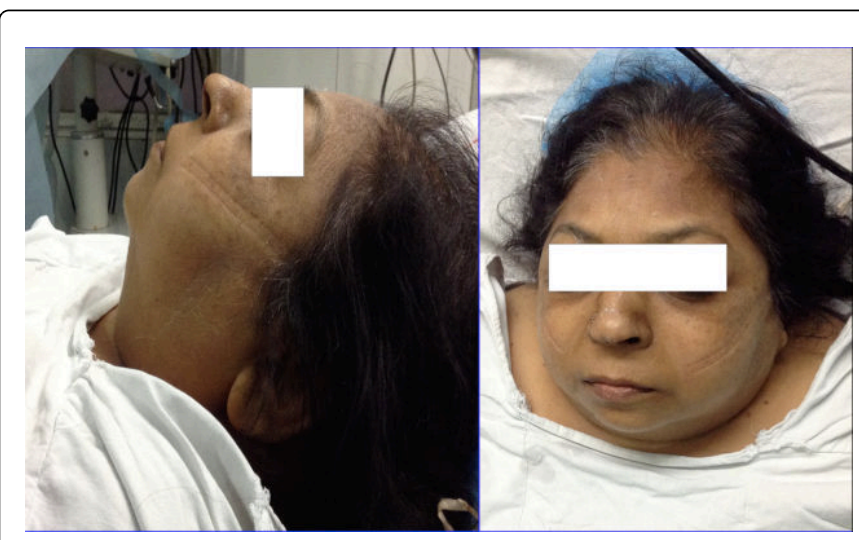

Figure 1: Achondroplastic feature of the patient showing difficult airway.

Foramen magnum is narrow and funnel shaped and can be compressed during laryngoscopy and cause cord ischemia leading to sudden death [4]. Pectus excavatum and kyphoscoliosis [6] of the thoracic spine causes restrictive lung disease resulting in decreased FRC, increase closing volume, atelectasis and on long term obstructive and central sleep apnoea, cor pulmonale and increase postoperative pneumonia [3].

Our patient had multiple features of predicted difficult airway with fusion of C3-C5 cervical spine and narrowed foramen magnum and hence we wanted to preserve the spontaneous respiration without any neck movement. Severe pulmonary disease predicted decrease FRC and rapid desaturation in case ventilation or intubation fail and usage of a laryngeal mask airway (LMA) as a rescue airway device may not be fruitful in this case due to the large tongue, decrease submandibular space and stiff lungs.

C-spine radiographs are suggested for preoperative airway assessment and predicting difficult intubation [4]. Difficulty in mask ventilation and impossible visualization of larynx $[7,8]$ are reported and smaller endotracheal tubes may be required due to laryngeal hypoplasia $[1,2]$.
In our case, effective patient counselling and planning, airway preparation and positioning help in the successful awake intubation in first attempt maintaining the neck stability throughout.

Although there are multiple reports using neuraxial anaesthesia, vertebral abnormalities can complicate its technique. Spinal anomalies include kyphoscoliosis, lumbar hyperlordosis, narrowing of vertebral canal, spinal cord stenosis and ischemia, short pedicles and reduced interpedicular distance and osteophyte formation $[4,9,10]$. Dry tap on subarachnoid block6 and narrow epidural space causing difficulty in threading catheter [10] has been reported. In our patient, kyphoscoliosis, lumbar hyperlordosis with degenerative changes with spinal fusion and difficulty in positioning of the patient due to limited hip flexion prompt us to avoid regional anaesthesia and hence was not considered for our anaesthesia technique.

Difficult intravenous access (IV) is usually present due to the joint deformity, increase subcutaneous fat and laxity of skin [4]. In our patient, intravenous access was difficult and we secured two large IV lines one before induction and the other under anaesthesia. Due to the short neck and restricted neck movement, central vein cannulation can be challenging in these patients, although it was not required in our patient as two large peripheral IV lines were secured. In view of the conical, short and fat arms, right radial artery was cannulated for invasive blood pressure monitoring. Due to presence of multiple osteophytes, immature bones and obesity leading to difficult surgical plane the intraoperative blood lost was more then usual and patient became hemodynamically unstable and hence only unilateral hip replacement was done. Patient was not extubated due to her poor chest condition preoperatively, difficult intubation and increase blood loss and was shifted to the ICU for postoperative management and the course was uneventful.

In summary, we emphasize the risk of neurological injury while extending the neck during laryngoscopy for tracheal intubation due to the anatomical abnormalities in these patients. The possibility of difficult mask ventilation and intubation should be always kept in mind and difficult airway cart should be kept ready to avoid airway catastrophe in these patients. A detail pre-anaesthetic evaluation and planning is utmost important in such patients and the anesthetic technique has to be individualized based on the patients anatomical characteristics and associated co-morbidities.

\section{References}

1. Banu Cevik, Serhan Colakoglu (2010) Anesthetic management of achondroplastic dwarf undergoing caesarean section. Mej Anesth 20: 907-910.

2. Francomano CA (1995) The genetic basis of dwarfism. N Engl J Med 332: 58-59.

3. Abrao MA, Silveira VG, CFLV Barcellos (2009) Anaesthesia for bariatric surgery in an achondroplastic dwarf with morbid Obesity. Rev Bras Anestesiol 59: 79-86.

4. R Gorji, R Nastasi, S Stuart, R Tallarico, F Li (2010) Anaesthesia and neuromonitoring for correction of thoracolumbar deformity in an achondroplastic dwarf. Internet J Anesthesiol 29: 1.

5. Sisk EA, Heatley DG, Borowski BJ (1999) Obstructive sleep apnea in children with achondroplasia -surgical and anesthetic considerations. J of Oto Head and Neck 120: 248-254.

6. Asmita P. Karnalkar, Ashok Deshpande (2015) Anaesthesia management of patient with achondroplasia for abdominal hysterectomy. Int J Sci Rep 1: 264-266.

7. Kalla GN, Fening E (1986) Anaesthetic management of achondroplasia. Br J Anaesth 58: 117-119. 
Citation: Neisevilie Nisa, Puneet Khanna, Dhruv Jain (2016) Anaesthetic Management of an Achondroplastic Dwarf with Difficult Airway and Spine for Total Hip Replacement: A Case Report. Gen Med (Los Angeles) 4: 227. doi:10.4172/2327-5146.1000227

Page 3 of 3

8. Monedero P, Garcia-Pedrajas F, Coca I (1997) Is management of anaesthesia in achondroplastic dwarfs really a challenge? J Clin Anesth 9: 208-212.

9. Hunter AGH, Bankier A, Rogers JG (1998) Medical complications of achondroplasia: A multicentre patient review. J Med Genet 35: 705-712.
10. Berkowitz ID, Raja SN, Bendor KS, Kopits SE (1990) Dwarfs: Pathophysiology and anesthetic implications. Anesthesiology 73: 739-759. 